\title{
Síndrome de Turner - desfecho de uma gestação espontânea
}

\section{Turner syndrome - outcome of a spontaneous pregnancy}

Carla Antoniana Ferreira de Almeida Vieira ${ }^{1}$. Eveline Gadelha Pereira Fontenele ${ }^{2}$. Áurea Maíla Albuquerque ${ }^{3}$ Rosana Quezado ${ }^{4}$. Ângela Delmira Nunes Mendes 5 . Rejane Araújo Magalhães ${ }^{6}$.

1 Médica, especialista em Clínica Médica, residência em Endocrinologia, Hospital Universitário Walter Cantídio (HUWC), Universidade Federal do Ceará (UFC), Fortaleza, Ceará, Brasil. 2 Médica, Endocrinologista, Doutorado em Biotecnologia, professora adjunta do Departamento de Medicina Clínica da Universidade Federal do Ceará (UFC), Fortaleza, Ceará, Brasil. 3 Médica, especialista em Clínica Médica, residente em Endocrinologia, Hospital Universitário Walter Cantídio (HUWC), Universidade Federal do Ceará (UFC), Fortaleza, Ceará, Brasil. 4 Médica, Endocrinologista, Mestrado em Ciências Médicocirúrgicas, Preceptora do serviço de Endocrinologia da Universidade Federal do Ceará (UFC), Fortaleza, Ceará, Brasil. 5 Médica, Endocrinologista, Mestrado em Ciências Médicas, Preceptora do serviço de Endocrinologia da Universidade Federal do Ceará (UFC), Fortaleza, Ceará, Brasil. 6 Médica, Endocrinologista, Mestranda em Patologia, Preceptora do serviço de Endocrinologia da Universidade Federal do Ceará (UFC), Fortaleza, Ceará, Brasil.

\section{RESUMO}

Objetivo: a síndrome de Turner afeta aproximadamente 1:2.500 meninas nascidas vivas e decorre da ausência completa ou parcial do segundo cromossomo X. Embora o hipogonadismo e a infertilidade sejam os achados mais frequentes, $2-5 \%$ das mulheres podem engravidar naturalmente. O objetivo deste trabalho é relatar o desfecho de uma gravidez espontânea em portadora de síndrome de Turner e apresentar uma revisão sobre o manejo dessa associação incomum. Relato do caso: paciente com síndrome de Turner diagnosticada aos 8 anos, cariótipo 45,X(4\%)/46,XX(96\%), apresentou desenvolvimento puberal espontâneo e menarca aos 15 anos. Aos 28 anos engravidou espontaneamente. Na $32^{\mathrm{a}}$ semana de gestação, ultrassonografia revelou hidropsia fetal, placentomegalia e polidramnia. A gestação evoluiu para trabalho de parto prematuro, com óbito do recém-nascido após 3 horas de vida. A paciente desenvolveu sintomas de depressão pós-parto, sendo encaminhada para acompanhamento psicológico. Conclusão: a gestação espontânea em uma mulher com síndrome de Turner é um evento raro. Há aumento de risco de malformações fetais, abortos espontâneos e natimortos, uma frequência elevada de complicações maternas e alta taxa de mortalidade. Complicações maternas mais comuns são diabetes gestacional, hipotireoidismo, hipertensão, pré-eclâmpsia, eclâmpsia e risco de dissecção/ ruptura da aorta, sendo fundamental acompanhamento multidisciplinar pré-concepcional e pré-natal.

Palavras-chave: Síndrome de Turner. Gravidez de alto risco. Complicações na gravidez.

\section{ABSTRACT}

Objectives: Turner syndrome affects approximately one in 2500 live-born females and results from the complete or partial absence of the second sex chromosome. Although hypogonadism and infertility are the most frequent features, $2-5 \%$ may present spontaneous pregnancy. The purpose of this paper is to report the outcome of a spontaneous pregnancy in a patient with Turner syndrome and to review management of this unusual association. Case report: the patient diagnosed with Turner syndrome at 8 years old, with $45, \mathrm{X}(4 \%) / 46, \mathrm{XX}(96 \%)$ karyotype, had spontaneous pubertal development and menarche at 15 years old. Spontaneous pregnancy occurred at age 28 and at the 32nd week of gestation ultrasound evaluation revealed hydrops fetalis, placentomegaly and polyhydramnios. The patient developed complications, including preterm birth and newborn death after three hours. Later she presented with postpartum depression. Conclusion: spontaneous pregnancy in woman with Turner syndrome is a rare event. There is an increased risk of chromosomal abnormalities, miscarriages and stillbirths, as well as an increased frequency of complications during pregnancy and higher mortality. The most common complications are gestational diabetes, hypothyroidism, hypertension, preeclampsia, eclampsia and increased risk of aortic dissection/rupture. These life-threatening conditions warrant multidisciplinary counseling prior to conception and during the prenatal period.

Keywords: Turner syndrome. Pregnancy, high-risk. Pregnancy complications.

Autor correspondente: Carla Antoniana Ferreira de Almeida Vieira, Rua Professor Carvalho, 2950, apartamento 106, Joaquim Távora, Fortaleza, Ceará. CEP: 60120-205.Telefone: +55 85 99973-7618. E-mail: carlaantoniana@yahoo.com.br

Conflito de interesses: Não há qualquer conflito de interesses por parte de qualquer um dos autores.

Recebido em: 27 Dez 2016; Revisado em: 10 Fev 2017; Aceito em: 06 Mar 2017. 


\section{INTRODUÇÃO}

A síndrome de Turner (ST) afeta aproximadamente 1:2.500 meninas nascidas vivas. As características mais comuns da síndrome são baixa estatura e falência ovariana. No período pré-natal, alterações, como presença de higromas císticos, coarctação da aorta, defeitos cardíacos, anormalidades renais, polidrâmnio ou oligodrâmnio e retardo no crescimento, podem ser detectadas. ${ }^{1}$ ST deve ser suspeitada em qualquer menina recém-nascida que apresenta edema em mãos e pés ou alterações cardíacas. ${ }^{2}$ Outras características fenotípicas incluem anormalidades esqueléticas, como cúbito valgo, membros curtos, genu valgo e quarto metacarpo curto, otite média crônica, perda auditiva, orelhas proeminentes e com implantação baixa, linfedema, epicanto, micrognatia, palato ogival, pescoço alado, cabelos em tridente e anormalidades renais. Complicações cardiovasculares incluem válvula aórtica bicúspide (VAB), coarctação da aorta, drenagem venosa anômala, prolapso de válvula mitral, hipertensão, dilatação da raiz da aorta e doença isquêmica. ${ }^{3}$ Cerca de 5 a $50 \%$ das pacientes apresentam malformações cardiovasculares. ${ }^{4}$

O diagnóstico de ST se baseia no julgamento clínico e deve ser considerado em paciente do sexo feminino com baixa estatura, associada ou não a falência ou atraso puberal, ou algum dos achados fenotípicos. A análise cromossômica é necessária para estabelecer o diagnóstico, pois esta condição é decorrente da ausência completa ou parcial do segundo cromossomo X. A análise padrão recomendada é de 30 células, identificando pelo menos $10 \%$ de mosaicismo com $95 \%$ de confiança. ${ }^{1} \mathrm{~A}$ maioria dos casos apresenta monossomia $(45, \mathrm{X})$, seguida por mosaicismo $(45, \mathrm{X} / 46, \mathrm{XX})$ com ou sem alterações estruturais no $\mathrm{X}$, tais como isocromossomo do braço longo (46,Xi(Xq)), deleções do braço curto (46,XdelXp22.3) ou do braço longo (45,X/46XdelXq2), translocações (46,X,t(X;15) ou cromossomo $\mathrm{X}$ em anel $(45, \mathrm{X} / 46, \mathrm{Xr}(\mathrm{X})) .{ }^{2} \mathrm{O}$ fragmento do cromossomo $\mathrm{Y}$ pode ser detectado em alguns casos. ${ }^{5}$
A maioria das pacientes com ST apresenta acelerada atresia folicular, com depleção dos folículos ovarianos ao nascer ou nos primeiros meses de vida. ${ }^{6}$ Entretanto, algumas podem apresentar puberdade espontânea, com ciclos menstruais regulares ou oligomenorréia, que em geral evoluem para falência ovariana precoce (FOP), com um período limitado para ocorrência natural de uma gestação. ${ }^{7}$ Assim, embora o hipogonadismo primário e a infertilidade sejam os achados mais frequentes, $2-5 \%$ das mulheres podem apresentar gravidez espontânea. ${ }^{8}$ No Brasil, não há ainda dados estatísticos sobre a prevalência de gravidez em pacientes com ST.

\section{RELATO DO CASO}

Paciente, sexo feminino, 28 anos, procedente de Fortaleza, teve o diagnóstico de ST aos 8 anos de idade, com cariótipo 45,X $(4 \%) / 46, \mathrm{XX}(96 \%)$, ao procurar assistência médica em outro serviço público de endocrinologia do estado, por baixa estatura. Fez uso de somatropina dos 9 aos 15 anos para promoção do crescimento. Teve desenvolvimento puberal espontâneo e menarca aos 15 anos, após o qual abandonou seguimento.

Em maio de 2015, aos 28 anos, a paciente engravidou espontaneamente. Foi sua primeira gestação. Com idade gestacional de 24 semanas, em virtude de aumento do TSH (4,0 mUI/L), foi encaminhada pelo serviço de obstetrícia ao serviço de endocrinologia de nossa instituição, sendo iniciado levotiroxina $50 \mathrm{mcg} / \mathrm{dia}$.

$\mathrm{Na} 32^{\mathrm{a}}$ semana de gestação, ultrassonografia obstétrica revelou feto masculino, com hidropsia, derrame pleural bilateral, ascite moderada, placentomegalia e polidramnia (Figura 1). Embora a paciente não tenha desenvolvido diabetes gestacional ou doença hipertensiva, a gestação evoluiu para trabalho de parto prematuro, por via cesárea, em virtude das complicações fetais.

Figura 1. US obstétrico evidenciando feto com derrame pleural bilateral (seta vermelha).

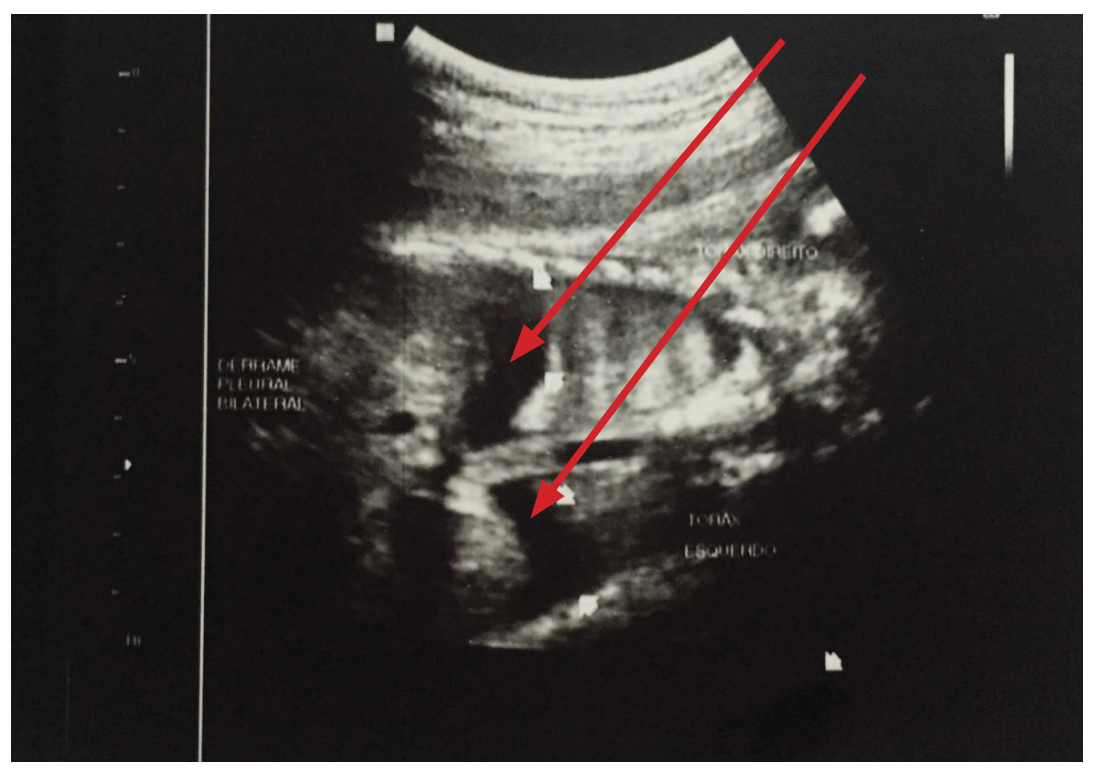


O recém-nascido apresentava-se em estado grave, hidrópico, com peso de 3635 gramas, $42 \mathrm{~cm}$ de estatura, hipotônico, sem respiração espontânea, frequência cardíaca de 110 batimentos/minuto, sendo encaminhado para a unidade de terapia intensiva neonatal. Evoluiu para óbito após 3 horas. A paciente desenvolveu sintomas de depressão pós-parto diante do desfecho distante das suas expectativas.

\section{DISCUSSÃO}

A gestação espontânea em uma mulher com ST é um evento raro. ${ }^{9}$ Pacientes desenvolvem FOP, não sendo detectável qualquer função ovariana na maioria dos casos. Cerca de $33 \%$ das meninas vão desenvolver naturalmente alguns sinais puberais, $10 \%$ completarão a puberdade e somente $2 \%$ apresentarão uma gravidez espontânea. ${ }^{10}$ Embora esta ocorra independente do cariótipo, é mais observada naquelas com alteração estrutural do X ou mosaicismo. ${ }^{6,9}$

A gestação em pacientes com ST está relacionada com risco materno-fetal elevado. Anormalidades cromossômicas podem ser detectadas no concepto (trissomia do 21, ST). ${ }^{10} \mathrm{Um}$ estudo de 160 gestantes com ST observou que dos 58\% nascidos vivos, $34 \%$ apresentaram malformação fetal por anormalidades cromossômicas. ${ }^{11}$ Existe também um risco maior de abortos espontâneos e natimortos do que na população geral..$^{5}$ Há uma maior frequência de complicações durante a gestação, com aumento em 100 vezes do risco de morte materna. ${ }^{12} \mathrm{~A}$ gestação per se impõe um aumento de estresse na aorta, pois mesmo mulheres multíparas normais têm dilatação da aorta ascendente quando comparadas ao grupo controle da mesma idade sem filhos. ${ }^{13}$ Devido a anomalias cardiovasculares inerentes associadas a ST, essas mulheres estão em maior risco de morrer por dissecção/ruptura da aorta e têm risco elevado de hipertensão induzida pela gravidez, pré-eclâmpsia, eclâmpsia, e suas complicações. Esse risco é maior no final da gestação ou no puerpério e em gestações múltiplas, que podem ocorrer por reprodução assistida..$^{9,10} \mathrm{O}$ risco de morte durante o período perinatal por dissecção ou ruptura de aorta em uma mulher com ST é de aproximadamente $2 \%{ }^{4}$

O risco de diabetes gestacional e de hipotireoidismo é elevado. Estas condições aumentam risco de hipertensão induzida pela gravidez e resultados adversos materno-fetais. ${ }^{10} \mathrm{Um}$ estudo sueco que avaliou 124 pacientes com ST que tiveram filhos, mostrou um aumento da incidência de doença tireoidiana comparada com aquelas com ST que nunca gestaram. Isso pode ser atribuído ao maior rastreamento de disfunção tireoidiana na gravidez ou na avaliação de infertilidade. ${ }^{14}$

Um estudo de coorte na França avaliou 480 pacientes com $\mathrm{ST}$, e a prevalência de gestação espontânea foi de apenas 5,6\%, com alta taxa de abortos espontâneos, 30,8\% versus $15 \%$ na população geral da mesma região. Os dados também mostraram maior taxa de anormalidades cromossômicas. Não houve diferença nos distúrbios tireoidianos autoimunes entre grávidas e não grávidas. A taxa de doença hipertensiva induzida pela gestação foi de $13,3 \%$, o dobro da população geral, mas não foi relatado nenhum caso de dissecção de aorta. ${ }^{15}$
A abordagem de pacientes com ST que apresentam puberdade espontânea, deve incluir medidas educativas visando prevenção e alerta sobre os riscos que uma gestação não planejada pode trazer. ${ }^{6}$ As pacientes e seus familiares devem ser informados dos riscos antes da decisão pela gestação. ${ }^{13}$ Uma vez decidido pela gravidez, o acompanhamento multidisciplinar por obstetra, cardiologista, e endocrinologista, durante todo o período perinatal é fundamental. O envolvimento de outros especialistas pode ser necessário, como nefrologistas e hepatologistas. $^{7}$

A avaliação pré-concepcional do risco cardiovascular inclui monitoramento da pressão arterial, eletrocardiograma, ecocardiograma e ressonância nuclear magnética cardíaca/ aórtica (RNM). ${ }^{9}$ O diâmetro da aorta ajustado para a superfície corpórea pode ser considerado um preditor de risco. ${ }^{4}$

Função tireoidiana, glicemia de jejum, hemoglobina glicada, função hepática, ultrassonografia renal e ginecológica devem ser também avaliados previamente à concepção para identificar fatores de riscos adicionais que podem aumentar ainda mais o risco de complicações materno-fetais. ${ }^{9,10}$

Embora a presença de VAB não contraindique a gravidez, ela é considerada um fator de risco. Por outro lado, história de cirurgia de aorta, dilatação de aorta acima de $35 \mathrm{~mm}$ ou $25 \mathrm{~mm} /$ $\mathrm{m}^{2}$, dissecção ou coarctação de aorta, hipertensão resistente ao tratamento e hipertensão portal com varizes de esôfago são consideradas contraindicações absolutas para gestação. ${ }^{9}$ Portanto, ST pode ser considerada uma contraindicação relativa para gravidez, independente dos fatores de riscos adicionais. $^{10}$

No pré-natal, a função cardíaca deve ser monitorada com ecocardiograma ao final do primeiro e do segundo trimestre, depois mensalmente, no terceiro trimestre. ${ }^{9,15}$ Um aumento do diâmetro aórtico superior ou igual a $10 \%$ entre dois exames deve ser confirmado por RNM. ${ }^{9} \mathrm{O}$ reconhecimento imediato da dissecção pode promover a oportunidade de uma intervenção cirúrgica bem sucedida. ${ }^{4}$

Além disso, durante a gestação também deve-se monitorar pressão arterial, peso e proteinúria. Essas pacientes tem aumento das taxas de cesárea, principalmente por desproporção feto-pélvica ou por complicações clínicas. ${ }^{7}$ Considerando que essas pacientes tem predisposição a aortopatia, podendo aumentar durante a gravidez ou o parto, o uso da cesárea como via de parto é razoável. ${ }^{6}$

No presente relato, uma paciente com ST teve gestação espontânea, e conforme mostra a literatura, evoluiu com complicações fetais. A paciente apresentada não realizou uma avaliação pré-concepcional, com o objetivo de avaliar o risco cardiovascular e receber orientações sobre os riscos materno-fetais. Além disso, a paciente teve um acesso tardio a uma unidade terciária, já no segundo trimestre. O óbito fetal foi um desfecho da gravidez muito distante das expectativas da paciente e da família, que evolui com quadro depressivo necessitando acompanhamento psicoterápico. 
É fundamental que pacientes com ST sejam seguidas continuamente por equipe interdisciplinar por toda vida, mesmo quando apresentam puberdade espontânea. A gestação em portadoras de ST deve ser considerada de alto risco. ${ }^{14} \mathrm{~A}$ equipe médica deve estar ciente da necessidade de rastrear defeitos cardiovasculares congênitos em mulheres com ST

\section{REFERÊNCIAS}

1. Bondy CA; Turner Syndrome Study Group. Care of girls and women with Turner syndrome: a guideline of the Turner Syndrome Study Group. J Clin Endocrinol Metab. 2007;92(1):10-25.

2. Sybert VP, McCauley E. Turner's syndrome. N Engl J Med. 2004;351(12):1227-38.

3. Castelo-Branco C. Management of Turner syndrome in adult life and beyond. Maturitas. 2014;79(4):471-5.

4. Practice Committee of American Society for Reproductive Medicine. Increased maternal cardiovascular mortality associated with pregnancy in women with Turner syndrome. Fertil Steril. 2012;97(2):282-4.

5. Doğer E, Çakıroğlu Y, Ceylan Y, Ulak E, Özdamar O, Çalışkan E. Reproductive and obstetric outcomes in mosaic Turner's syndrome: a cross-sectional study and review of the literature. Reprod Biol Endocrinol. 2015;13(59):1-7.

6. Hadnott TN, Gould HN, Gharib AM, Bondy CA. Outcomes of spontaneous and assisted pregnancies in Turner syndrome: the US National Institutes of Health experience. Fertil Steril. 2011;95(7):2251-6.

7. Bouet PE, Godbout A, El Hachem H, Lefebvre M, Bérubé L, Dionne MD, et al. Fertility and pregnancy in Turner syndrome. J Obstet Gynaecol Can. 2016;38(8):712-8.

8. Hovatta O, Hreinsson J, Fridström M, Borgström B. Fertility and pregnancy aspects in Turner syndrome. International Congress Series. 2006;1298:185-189. assintomáticas que planejam uma gravidez. ${ }^{13}$ A adoção deve ser considerada como uma alternativa para aquelas que almejam a maternidade. ${ }^{12} \mathrm{~A}$ equipe deve proporcionar àquelas pacientes que desejem gestar, uma minuciosa avaliação dos riscos e aconselhamento genético antes da concepção, além de rigoroso seguimento perinatal.

9. Cabanes L, Chalas C, Christin-Maitre S, Donadille B, Felten ML, Gaxotte V, et al. Turner syndrome and pregnancy: clinical practice. Recommendations for the management of patients with Turner syndrome before and during pregnancy. Eur J Obstet Gynecol Reprod Biol. 2010;152(1):18-24.

10. Karnis MF. Fertility, pregnancy, and medical management of Turner syndrome in the reproductive years. Fertil Steril. 2012;98(4):787-91.

11. Tarani L, Lampariello S, Raguso G, Colloridi F, Pucarelli I, Pasquino AM, et al. Pregnancy in patients with Turner's syndrome: six new cases and review of literature. Gynecol Endocrinol. 1998;12(2):83-7.

12. Grynberg M, Bidet M, Benard J, Poulain M, Sonigo C, CédrinDurnerin I, et al. Fertility preservation in Turner syndrome. Fertil Steril. 2016;105(1):13-19.

13. Bondy C. Pregnancy and cardiovascular risk for women with Turner syndrome. Womens Health (Lond). 2014;10(4):469-76.

14. Hagman A, Kallen K, Bryman I, Landin-Wilhelmsen K, Barrena ML, Wennerholm UB. Morbidity and mortality after childbirth in women with Turner karyotype. Hum Reprod. 2013;28(7):1961-73.

15. Bernard V, Donadille B, Zenaty D, Courtillot C, Salenave S, Brac de la Perrière A, et al. Spontaneous fertility and pregnancy outcomes amongst 480 women with Turner syndrome. Hum Reprod. 2016;31(4):782-8.

\section{Como citar:}

Vieira CA, Fontenele EG, Albuquerque AM, Quezado R, Mendes AD, Magalhães RA. Síndrome de Turner - desfecho de uma gestação espontânea. Rev Med UFC. 2017 mai-ago;57(2):69-72. 